\title{
Les ambitions documentaires et didactiques de la description du métier de pêcheur dans le Traité des Pêches
}

Maryvonne Merri et Audrey Doualot

\section{(2) OpenEdition}

Journals

Édition électronique

URL : https://journals.openedition.org/educationdidactique/1794

DOI : 10.4000/educationdidactique. 1794

ISSN : 2111-4838

Éditeur

Presses universitaires de Rennes

Édition imprimée

Date de publication : 31 décembre 2013

ISBN : 978-2-7535-3327-1

ISSN : $1956-3485$

Référence électronique

Maryvonne Merri et Audrey Doualot, «Les ambitions documentaires et didactiques de la description du métier de pêcheur dans le Traité des Pêches », Éducation et didactique [En ligne], 7-3 | 2013, mis en ligne le 31 décembre 2015, consulté le 16 août 2022. URL : http://journals.openedition.org/ educationdidactique/1794; DOI : https://doi.org/10.4000/educationdidactique.1794 


\title{
LES AMBITIONS DOCUMENTAIRES ET DIDACTIQUES DE LA DESCRIPTION DU MÉTIER DE PÊCHEUR DANS LE TRAITÉ DES PÊCHES
}

\author{
Maryvonne Merri, Département de psychologie, Université du Québec à Montréal
} Audrey Doualot, Département de psychologie, Université du Québec à Montréal

Le Traité des Pêches, publié au XVIII ${ }^{\mathrm{e}}$ siècle par Duhamel du Monceau dans le cadre du projet de Description des arts et métiers demeure une œuvre de référence. Elle poursuit, en effet, une ambition à la fois documentaire et didactique. Cet article analyse les cadres philosophiques, politiques de ce traité. Il montre que l'enseignement de la pêche est justifié pour Duhamel du Monceau par la préservation de la ressource et la rentabilité économique. Enfin, cet article caractérise les procédés utilisés par Duhamel du Monceau pour réaliser son ambition d'enseignement des techniques recueillies auprès des pêcheurs.

Mots-clés: Traité des Pêches, Source imprimée ancienne, Didactique de la pêche, Procédés didactiques, documentation

The Documentary and Didactic Ambitions of the Description of the Fishing Trade in the Traité des Pêches

The Traité des Pêches, published in the eighteenth century by Duhamel du Monceau in the Description des arts et métiers remains a work of reference. Indeed, it pursues both a documentary and didactic ambition. This paper analyzes the philosophical and political framework of the treatise. It shows how resource conservation and economic efficiency justify Duhamel de Monceau's teaching of fishing. Furthermore, this article characterizes the instructional processes Duhamel de Monceau used to achieve his ambition of teaching the techniques acquired from the fishermen.

Keywords: Traité des Pêches, Old printed source, Teaching Fishing, Instructional processes, Documentation 


\section{INTRODUCTION}

Au moment où Diderot et d'Alembert publient le Dictionnaire raisonné des sciences, des arts et des métiers ${ }^{1}$, Duhamel du Monceau (1700-1782) dirige le projet monumental de Description des Arts et Métiers de l'Académie des Sciences ${ }^{2}$. Le Traité des Pêches et Histoire des Poissons ${ }^{3}$, objet de cet article, a été rédigé par Duhamel du Monceau. Nous étudierons plus particulièrement le premier chapitre de la section relative aux filets de pêche (Duhamel du Monceau, 1776 , p. 155-200)..

Pourquoi étudier un texte du XVIII ${ }^{e}$ siècle? En premier lieu, une partie des gestes relatifs au filet décrits dans le Traité des Pêches restent exercés par les pêcheurs actuels en particulier les gestes de " ramendage " c'est-à-dire de réparation des filets ayant subi des accrocs. De plus, de nombreux engins de pêche du XX ${ }^{\mathrm{e}}$ et du XXI ${ }^{\mathrm{e}}$ siècles ont une origine ancienne (Deschamps, 2009 ; Perpillou, 1946). La réédition de ce traité en $1998^{4}$, au-delà de son intérêt de documentation historique, offre donc un éclairage des pratiques et instruments contemporains pour les enseignants et techniciens de pêche ${ }^{5}$.

En deuxième lieu, Duhamel du Monceau et les Académiciens proposent un discours sur les techniques ou technologie pour formaliser les discours artisanaux (Carnino, 2010). Ce projet est affirmé dans le titre Description des Arts et Métiers. Réaumur, directeur de ce projet avant Duhamel du Monceau, définit ce projet dans sa préface inédite:

L'histoire des Arts que nous avons entrepris d'écrire n'est point celle de leur progrès, de leur décadence, mais celle des pratiques qui sont actuellement en usage; nous avons appelé cette espèce de description « histoire des Arts », comme on nomme « histoire naturelle » la description des productions de la nature (Réaumur, cité dans Jaoul \& Pinault, 1982).

Le Traité des Pêches est une tentative de mettre en forme les savoirs des pêcheurs et de rationnaliser l'activité de ceux-ci. Pour cela, Duhamel du Monceau introduit différents registres de technicité (BrièreGuenoun, Perez, \& Durey, 2007 ; Martinand, 1994). En effet, Duhamel du Monceau donne au lecteur non seulement les conditions pour maîtriser les techniques mais aussi pour les interpréter. Il propose à la fois une description " exacte $^{6} »$ des techniques recueillies auprès des pêcheurs dans les ports et une documentation du contexte juridique, social et économique de l'époque.

Enfin, la Description des Arts et Métiers contribue au développement économique et technique du pays par la diffusion écrite des techniques. Duhamel du Monceau met alors en place des procédés d'enseignement fondés sur l'écrit, tentative nouvelle dans un métier à transmission gestuelle et orale.

Cet article a pour objectif d'étudier une partie du Traité des Pêches selon les trois cadres imbriqués qui en définissent l'intention et la mise en œuvre: au premier niveau, la volonté politique et les idées philosophiques, au deuxième niveau, l'ambition de documentation de l'activité de la pêche et au troisième niveau, le choix de procédés didactiques susceptibles de diffuser les techniques relatives au filet de pêche par l'écrit et les figures. Ces trois niveaux seront étudiés successivement après une présentation de la structure du texte étudié.

\section{LA STRUCTURE ET LE CONTENU DU TEXTE}

Le tome $\mathrm{V}$ de la deuxième édition de la Description des Arts et Métiers contient Les trois premières sections du traité des pêches E l'histoire des poissons. La deuxième section de ce tome s'intitule De la pêche au filet ${ }^{7}$. Celle-ci peut être lue indépendamment de la section précédente sur les hameçons.

Le texte étudié dans cet article correspond au chapitre premier de cette seconde section sur les filets $\left(\mathrm{TP}^{8}\right)$. Il porte sur Des filets, de leur fabrique, de leur entretien, E leurs différentes espèces. Il est divisé en une introduction générale à la section sur les filets de pêche, puis pour le chapitre I, en une introduction, quatorze articles et une récapitulation. L'ordre d'exposé est caractéristique des projets encyclopédiques du XVIII ${ }^{\mathrm{e}}$ siècle et est probablement conforme aux consignes laissées par Réaumur, premier directeur du projet de Description des Arts et Métiers (Dupont de Dinechin, 1999):

Son plan (de Duhamel) est toujours à peu près le même. Dans l'Avertissement ou l'Introduction, il indique ses principales sources d'information, rappelant notamment ce qu'il a trouvé dans les dossiers de Réaumur et les noms des personnes qu'il a consultées. Il décrit ensuite les matériaux ou matières premières en cause, les outils utilisés et les procédés mis en œuvre; dans certains cas, un lexique figure à la fin de l'ouvrage 
et fournit le vocabulaire technique qu'il est nécessaire de connaître (p. 344).

La représentation du travail comme action instrumentée sur la nature détermine l'ordre de description du métier, ordre commun à l'Encyclopédie et à la Description des Arts et Métiers: le matériau naturel est présenté puis les artefacts créés par l'homme et enfin les procédés de l'artisan. Ainsi, bien avant que la technologie comme étude et discours sur les techniques humaines n'existe comme science (Mauss, 2004 ; Vatin, 2004), les auteurs du XVIII ${ }^{\mathrm{e}}$ siècle ont développé des moyens de description des techniques humaines qui préfigurent le concept de chaîne opératoire développé par Mauss et Leroi-Gourhan (Martinon-Torres, 2002). Une chaîne opératoire est définie comme "l'ensemble ordonné de gestes individuels et collectifs mis en œuvre pour obtenir un objet. C'est une décomposition gestuelle du processus d'obtention du résultat. » (Garçon, 2006, p. 23)

Duhamel du Monceau présente donc dans l'article 1 le matériau naturel (le chanvre) qui permet de fabriquer les filets et il distingue les différents types de filets selon la taille des mailles.

L'article 2 décrit ensuite l'apprêt du matériau naturel, les types de mailles et les outils. Il présente la fabrication des fils et catégorise les filets (tailles et types de mailles), les outils du mailleur ${ }^{9}$, le vocabulaire relatif aux tâches du mailleur.

Les articles 3 à 8 présentent les techniques de fabrication des filets. Disposant du fil et des caractéristiques du filet, il s'agit à présent de passer à l'examen technique des tâches permettant de le réaliser: faire les nœuds, fabriquer les filets avec les mailles en losange, enlarmer et accroître le filet, diminuer le filet ${ }^{10}$, fabriquer les filets à mailles carrées, les filets de type trémail, les filets ronds, cylindriques, coniques, les filets ronds avec des goulets.

Si faire les nœuds, enlarmer, accroître, diminuer le filet sont des tâches et des techniques générales (articles 3 à 7), les articles 8 à 11 permettent de spécifier des techniques relatives aux différents types de mailles et aux différents types de filets.

Enfin, les articles 12 à 14 présentent les techniques requises par la mise à l'eau du filet et son usure: le raccommodage, le lest et la conservation des filets.

Le texte principal est associé à deux suppléments en fin de volume:
- Une Explication de plusieurs termes qui sont en usage entre les pêcheurs, E communément peu connus de ceux qui ne sont point occupés à la pêche est introduite par un texte la justifiant par la valeur et la variété régionale des termes de pêche:

Il n'y a point de science, d'art, même de métier, qui n'aient des termes qui leur sont propres; \& ceux qui veulent s'instruire de quelque science ou de quelque part, doivent commencer par apprendre la valeur $\&$ la vraie signification de ces termes, sans quoi ils ne pourront prendre une juste idée de ce qu'on leur expliquera avec le plus grand soin. [...] les côtes de la France étant fort étendues, \& les lieux où l'on pratique les différentes pêches, éloignés du centre du royaume, le langage des pêcheurs, qui est absolument ignoré dans les provinces éloignées de la mer, est de plus sujet à varier beaucoup sur les différentes côtes où l'on s'occupe de la pêche. (TP, seconde section, p. 105)

- Aux 14 articles sont associées cinq planches de figures qui représentent, pour la planche I, les différentes sortes de mailles et le filet à ses différentes étapes de construction; pour la planche II, l'amorce et le plan de construction du filet carré, les flotteurs et les bordures du filet; pour la planche III, les techniques des nœuds sur le pouce et sur sous le petit doigt; pour la planche IV, les différentes étapes de la réparation des filets; pour la planche $\mathrm{V}$, le filage et la torsion du fil et les instruments du pêcheur (aiguilles, rouet pour filer le chanvre...).

\section{LA VOlONTÉ POLITIQUE ET LES IDÉES PHILOSOPHIQUES}

\section{Une commande de l'État}

Les Académiciens des Sciences du XVIII ${ }^{\mathrm{e}}$ siècle poursuivent un projet commandité au siècle précédent par Colbert, contrôleur général des Finances de Louis XIV, promoteur du développement du commerce et de l'industrie par la création de manufactures et créateur de l'Académie Royale des Sciences (Briggs, 1991 ; Mafarette-Dayries, 2000). Celle-ci a une mission de développement artisanal et industriel par la diffusion des techniques et secrets de fabrication auparavant jalousement gardés par les corporations (Dupont de Dinechin, 1999). 
Réaumur est le premier directeur de la Description. Mais s'il rassemble de nombreuses informations, il ne trouvera jamais le temps de les exploiter, son œuvre tombant alors aux mains de personnes peu scrupuleuses. À la mort de Réaumur, Duhamel du Monceau prend en charge la direction de la Description des Arts et Métiers (Dupont de Dinechin, 1999).

L'objectif politique et économique du projet est la rationalisation du travail par la transmission écrite des techniques aux praticiens. Sur ce point, Duhamel du Monceau est influencé, comme Réaumur, par leur ami commun Trudaine, intendant des finances qui souhaite vulgariser les techniques auprès des professionnels:

Dans cette perspective, la Description [...] diffère donc profondément de l'Encyclopédie de Diderot et d'Alembert, qui se proposent de transmettre des idées métaphysiques à partir des connaissances pratiques qu'ils ont collectées (Dupont de Dinechin, 1999, p. 332).

\section{L'importante contribution de Duhamel du Monceau}

Les descriptions de métiers produites par les Académiciens et cordonnées par Duhamel du Monceau sont variées et Duhamel du Monceau en rédige lui-même de très nombreuses (PinaultSorensen, 2000): le charbonnier, la fabrication des ancres, le cirier, le cartier, le serrurier, l'art de faire les colles, l'amidonnier, le couvreur... :

De 1761 à 1788 paraissent donc 73 monographies réparties en 99 fascicules in-folio, sans compter le Traité des Pêches. L'ensemble de la collection compte 9950 pages et comprend 1626 planches gravées. [...] 20 monographies de la Description portent sa signature, associée dans deux cas à celle de Réaumur, dont il reprend l'Art de l'épinglier et la Fabrique des Ancres (Dupont de Dinechin, 1999, p. 343).

Parmi les descriptions, les métiers maritimes occupent une place importante pour Duhamel du Monceau, également Inspecteur Général de la Marine. Le Traité des Pêches correspond aux volumes $\mathrm{V}$ et $\mathrm{X}$ de la Description. Cet ouvrage comporte 1246 pages de texte et 229 planches de figures (Thibault, 2000).
Ce traité comporte trois parties: la première partie a pour co-auteur de la Marre et est consacrée aux techniques de pêche, en particulier aux hameçons et aux filets, au déchargement et à la conservation du poisson. Les deuxième et troisième parties, signées par Duhamel du Monceau seul, concernent différentes espèces de poissons.

Pour rédiger le Traité des Pêches, Duhamel du Monceau s'appuie, comme d'ailleurs Diderot ${ }^{11}$, sur le travail considérable mené par Le Masson du Parc au début du XVIII ${ }^{\mathrm{e}}$ siècle (Thibault, 2000) et qui n'a été publié partiellement que récemment (Le Masson du Parc, 2004, 2009). Homme de science, attaché à l'exactitude des faits relatés (Dupont de Dinechin, 1999), Duhamel du Monceau consacre tout son temps et d'importants moyens financiers personnels au recueil de données en se déplaçant luimême dans les ports ${ }^{12}$ en utilisant son réseau européen (Michaud, 2000) mais aussi celui des Antilles (Guadeloupe), d'Amérique (Canada) ou encore d'Asie Mineure (Allard, 1970), et en s'attachant la collaboration de ses neveux (Dupont de Dinechin, 1999).

Une reconnaissance sociale et intellectuelle du travail manuel

Ce traité est représentatif de l'intérêt des savants du XVIII ${ }^{e}$ siècle pour les arts mécaniques qui permettent de transformer la matière. En effet, les philosophes, et en particulier Rousseau, prônent l'utilité sociale et l'autonomie économique que procure le travail manuel (Artz, 1938 ; Vérillon, Leroux, \& Manneux, 2005). Dans son introduction à la section II sur la pêche au filet, Duhamel du Monceau avance d'emblée la contribution des pêcheurs au bien commun:

On a pu apercevoir [...] que les pêcheurs se livrent à des travaux très pénibles et qu'ils s'exposent à de grands dangers, pour fournir des aliments à ceux qui, occupés d'autres objets, se nourrissent en partie des produits de la pêche, sans avoir presqu'aucune idée des peines qu'il faut se donner pour prendre les poissons qu'on sert sur leurs tables. (TP, § 1).

Tout comme Diderot qui affirme dans l'article Art de l'Encyclopédie ${ }^{13}$ la valeur médiatrice de l'instrument entre la main et l'objet, Duhamel du Monceau 
Maryvonne Merri et Audrey Doualot

place l'instrument du pêcheur au cœur de la description du métier comme le montrent les intitulés des sections du Traité: "de la pêche aux hameçons ", "de la pêche au filet ». Dans l'extrait suivant, le filet apparaît être une évolution du panier ou de la toile pour enfermer, contenir et tirer à terre les poissons:

Il est assez naturel que ceux qui habitaient le bord des rivières et de la mer, apercevant un nombre de poissons rassemblés en un endroit, ont essayé de les couvrir avec des paniers, pour les empêcher de se disperser; ou bien ils ont essayé de passer ces paniers en dessous, afin d'enlever ces poissons hors de l'eau; ou encore ils ont essayé de les renfermer par des toiles ou d'autres tissus équivalents pour les tirer à terre. [...] Cette idée a dû conduire à celle des filets. (TP, § 1)

Ainsi, comme le signalent Vérillon, Leroux \& Manneux (2005), les savants des Lumières, dont Duhamel du Monceau, affirment un lien entre les processus orientés vers la production et les processus de pensée. La reconnaissance de l'activité manuelle s'exprime également dans le choix même d'une démarche de description. Décrire par écrit les instruments et les gestes professionnels permet d'abstraire ceux-ci et, ainsi, d'en faire des objets évaluables par la pensée. Duhamel du Monceau n'applique donc pas les lois de la science à la nature mais choisit d'effectuer le mouvement inverse aux « sciences de l'ingénieur » en décrivant précisément le geste humain pour une appréciation de celui-ci (Vérin, 2000) ${ }^{14}$ selon des catégories scientifiques, légales, ergonomiques et sociales.

\section{Les différents destinataires de l'œuvre}

Qui sont les destinataires du Traité? Il y a certes les pêcheurs eux-mêmes auxquels Duhamel du Monceau fait plusieurs fois référence au début de la section II relative aux filets de pêche: « Il faut que les pêcheurs soient instruits fort en détail de tout ce que nous venons d'indiquer $[\ldots]$ »

Et aussi à la fin de cette section: « Nous espérons que ce petit traité de la fabrique des filets, qui n'avait pas encore été donné avec exactitude, sera utile aux pêcheurs ».

Mais Duhamel du Monceau élargit immédiatement son lectoratà toute personne curieuse:
Un point de notre ouvrage qui sera bien digne de l'attention des personnes qui se plaisent à réfléchir, est l'exposé clair et détaillé de toutes les industries que les hommes ont imaginées pour saisir leur proie, allant la chercher jusqu'au fond des eaux [...]. (TP, p. 12)

Plus encore, Bertrand, éditeur de l'édition de Neuchâtel de la Description des Arts et Métiers affirme que les volumes édités sont des « lumières » tant pour les générations à venir que pour la génération présente:

D'ailleurs, cet ouvrage n'est pas seulement destiné à ce siècle éclairé, où les Arts commencent à être cultivés avec le soin qu'ils méritent. On a travaillé pour les races futures. [...] Ainsi nos neveux, avec ces descriptions seront en état de profiter de nos lumières, de juger du terme où demeureront les arts avec nous, de leur progrès jusqu'à eux, enfin de ce qui reste à faire pour les porter au degré de perfection, dont ils sont susceptibles (Bertrand, 1771 , p. VI).

La Description des Arts et Métiers en rompant à la fois une transmission des gestes professionnels fondée sur le respect des aînés, introduit une distinction entre savoir et exécution, entre tutelle technique et travail. L'éditeur Bertrand, sans doute également motivé par la recherche d'un marché éditorial, l'écrit sans détours dans sa préface générale aux volumes de la Description des Arts et Métiers:

Il serait fort utile sans doute que chaque ouvrier joignant quelque théorie à la pratique, pût lire la description de son art; mais il l'est encore plus que les Manufacturiers soient bien instruits des pratiques de celui qu'ils font exercer. [...] bientôt les ouvriers mieux dirigés travailleront avec plus d'intelligence [...] (Bertrand, 1771, p. VII).

Cette séparation entre conception et exécution est familière à Duhamel du Monceau qui, comme nous l'avons dit, est Inspecteur Général de la Marine et développe, dans ce cadre, le corps des Ingénieurs Constructeurs de la Marine. L'organisation du métier de la pêche ne permet pas encore la création d'un tel corps de conception technique ${ }^{15}$, faute d'être entrée dans une ère comparable à la production manufacturière. Il demeure, pourtant, que les pêcheurs qui étaient auparavant les destinataires exclusifs de la formation orale et gestuelle traditionnelle ne sont 
plus les seuls bénéficiaires des techniques de leur propre métier.

Comme le montre Chartier, les faibles taux d'alphabétisation ou la non possession de livres n'excluent pas une accessibilité à l'écrit sous l'Ancien Régime, en particulier par la lecture par autrui ou les bibliothèques. Aussi, en publiant les différents traités en volumes séparés, l'Académie tente de réduire le coût des ouvrages et les rendre ainsi accessibles à tous. C'est d'ailleurs également la préoccupation de l'éditeur Bertrand qui préface ainsi l'édition de Neuchâtel de 1771-1783, cherchant encore à réduire le prix des ouvrages:

Lédition de Paris [la première édition] est faite avec magnificence, je dirais même un luxe inutile, s'il n'y avait pas toujours des gens riches et des bibliothèques capables de payer cher un livre superbement imprimé. Mais cette édition coûteuse empêche que le livre ne soit assez répandu (Bertrand, 1771 , p. XIII).

Dans les faits, il est probable que peu nombreux furent les pêcheurs contemporains de Duhamel du Monceau qui accédèrent directement à son Traité tant en raison du coût de l'ouvrage que du recours à l'écrit. En effet, l'exposé écrit des techniques est en rupture avec la forme traditionnelle de l'apprentissage de la pêche, dont on peut supposer qu'elle était, au XVIII ${ }^{\mathrm{e}}$ siècle, celle encore observée au XX ${ }^{\mathrm{e}}$ siècle par Delbos et Jorion (Delbos \& Jorion, 1984) dans la petite pêche. "Apprendre c'est travailler » résument ces auteurs, observant que ce sont les tâches confiées progressivement au novice, les succès vécus et les réprimandes reçues qui vont lui permettre d'acquérir les compétences du pêcheur.

En définitive, le Traité des Pêches est surtout représentatif de l'ambition à la fois morale et intellectuelle de Duhamel du Monceau pour ses lecteurs potentiels, qu'ils soient ses contemporains ou ceux des siècles à venir. La transmission des techniques par l'écrit est pour les académiciens et encyclopédistes une ambition en soi, synonyme de progrès social et cognitif pour tous (Nonnon, 2007). L'œuvre est alors laissée aux pratiques de lecture variées de ses différents lecteurs, qu'il s'agisse pour certains de parfaire seuls des gestes qu'ils n'auraient pu acquérir auparavant que sous des formes orales, concrètes et vicariantes (Bandura, 1977), pour d'autres de transposer (Chevallard, 1985) les techniques auprès des pêcheurs, ou encore de s'informer par curiosité de l'organisation du métier de la pêche. Dans les faits, la démocratie est au principe même du Traité des Pêches, comme de toute œuvre encyclopédique du XVIII ${ }^{e}$ siècle: il s'agit de parler de "tout" à " tout le monde » en laissant le lecteur décider seul d'une pratique, même si le livre est indisponible tant économiquement que psychologiquement pour une grande partie de la population (Habermas, 1997 ; HilairePerez \& Thébaut-Sorger, 2006).

\section{Une ouvre documentaire et didactique}

Pour «parler de tout à tout le monde », le Traité des Pêches poursuit à la fois deux ambitions qui seront analysées dans la suite de cet article:

Une ambition documentaire: Pour rédiger le Traité des Pêches, Duhamel du Monceau a organisé, nous l'avons dit, l'importante documentation de Le Masson du Parc mais aussi celle rassemblée par ses soins, par ses collaborateurs et correspondants répartis en France et dans le monde. Dans les premiers chapitres de la section relative à la préparation du matériau, l'auteur situe le métier dans un contexte social, économique et légal. Cette description offre aux lecteurs un exposé de l'organisation et les problèmes de l'activité de la pêche au XVIII ${ }^{e}$ siècle qui pourrait motiver, à lui seul, la consultation du Traité des Pêches.

Une ambition didactique: Duhamel du Monceau entreprend de transmettre les principales techniques relatives aux filets de pêche avec l'ambition de rendre compétent le lecteur. Il met en œuvre un va-et-vient entre un discours écrit de description technique et des figures représentant les outils et filets du mailleur. Duhamel du Monceau emploie ici des procédés didactiques particuliers et adaptés à une transmission exclusivement écrite des techniques.

La suite du texte décrira et articulera ces deux ambitions de la section des filets de pêche.

\section{L'AMBITION DE DOCUMENTATION DE L'ACTIVITÉ DE LA PÊCHE}

La première ambition de Duhamel du Monceau est donc de produire une description documentée de l'activité de pêche. Duhamel expose le contexte de l'activité de fabrication, de réparation et d'entretien des filets dans les premiers paragraphes de 
la section 2 puis, plus ponctuellement, lors de la présentation de techniques précises, pour marquer, en particulier, l'importance de la division du travail et des compétences techniques pour la réduction des coûts. Il s'agit, en effet, de garantir la prospérité pour la population du littoral qui fournit de nombreuses recrues à la marine militaire, activité importante pour le pouvoir royal (Busson, 1888).

Duhamel du Monceau propose ici une description à la fois conservatrice et critique de l'activité de pêche. La division du travail ${ }^{16}$ familial selon les âges et selon les sexes, et la solidarité économique entre pêcheurs et consommateurs plus riches sont les fondements inamovibles de l'activité. Mais deux facteurs menacent la pérennité de la pêche. En premier lieu, les usages des instruments de pêche épuisent la ressource vivante et préoccupent le pouvoir royal. En second lieu, les coûts de fabrication des filets grèvent les bénéfices des pêcheurs.

\section{Une description de l'activité en partie conservatrice}

En distinguant les actions individuelles à la fois finies et circonscrites et une activité collective orientée vers un objet commun (Engeström \& Sannino, 2010 ; Leontiev, 1984), Duhamel du Monceau définit les maillons de la chaîne opératoire comme autant d'actions élémentaires réparties entre les membres de la communauté. Conformément au premier principe de séparation dans la division sexuelle du travail (Kergoat, 2000), certains travaux sont "pour " les hommes et d'autres "pour " les femmes. Les femmes et les filles ne sont pas représentées dans les planches I, II et III correspondant aux filets mais elles sont présentes, de manière secondaire, pour filer le chanvre, matière première des fils de pêche, et pour tordre le fil. Les femmes déchargent les hommes de certains travaux et constituent ainsi une main-d'œuvre supplémentaire conformément au second principe de la division sexuelle du travail, le principe hiérarchique: "un travail d'homme "vaut" plus qu'un travail de femme » (Kergoat, 2000, p. 35), comme la présence sélective des femmes sur les planches le met en évidence (Sheridan, 2009). Certains termes sont révélateurs chez Duhamel du Monceau d'une valorisation des actions de confection $\mathrm{du}$ filet relativement à la préparation de la matière première: les femmes peuvent "même " aider les hommes en étant « au moins aussi habiles » qu'eux dans le laçage et le maillage:

Quand ils ont une nombreuse famille, les femmes et les filles s'occupent à la (la filasse) filer. Mais comme la fabrique des filets exige beaucoup de main-d'œuvre, \& comme on peut pour un écu de fil faire une étendue de filet qui coûterait 12 livres, les pêcheurs, pour peu que leur famille soit nombreuse, travaillent eux-mêmes leurs filets; les femmes \& les filles retordent le fil, \& même aident aux hommes à lacer ou mailler les filets, étant au moins aussi habiles qu'eux à cette sorte de travail. (§ 10)

Les hommes et les femmes sont également distingués par leur force physique. Ainsi, les femmes tordent le fil utilisé pour le corps du filet tandis que les hommes confectionnent les ainards, petites cordes qui servent à attacher le filet aux ralingues ${ }^{17}$ qui les bordent:

Il n'est pas hors de propos de remarquer qu'il y a une grande différence entre les fils simplement doublés \& retors par les femmes, $\&$ ceux qui sont commis par l'homme (pl., fig. 2). Les femmes roulent l'un sur l'autre les deux fils qu'elles ont soin de tenir mouillés, les deux pelotes étant dans un vase rempli d'eau. [...] Il n'en est pas de même des fils que commet l'homme (fig. 2). [...] il faut une force plus considérable pour désunir ces fils commis, que ceux qui ont été simplement retors. (§ 24 et 25)

Tandis que les femmes restent tournées vers la sphère intérieure, les hommes entretiennent, quant à eux, des relations marchandes avec les vendeurs de lin ou de cordes. Les pêcheurs âgés et infirmes vendent des filets: "Il y a des pêcheurs âgés ou infirmes qui s'occupent avec leur famille à faire des filets qu'ils vendent à l'aune. » (§ 14)

L'activité du pêcheur est principalement justifiée dans le Traité des Pêches par sa pérennité économique et un bénéfice réciproque entre les classes sociales ${ }^{19}$ :

Les pêcheurs se livrent à des travaux très pénibles [...] Mais d'un autre côté, ce sont les consommateurs qui subviennent, par l'achat du poisson, à la subsistance des pêcheurs $\&$ aux grands frais de la pêche; car celles qui se font aux filets, exigent surtout des dépenses considérables. Le luxe des gens très opulents tourne donc très efficacement au profit des pêcheurs. ( $\S 1$ ) 
Document 1. Illustration du filage du chanvre et de la fabrication des ainards (première édition du Traité des Pêches) ${ }^{18}$

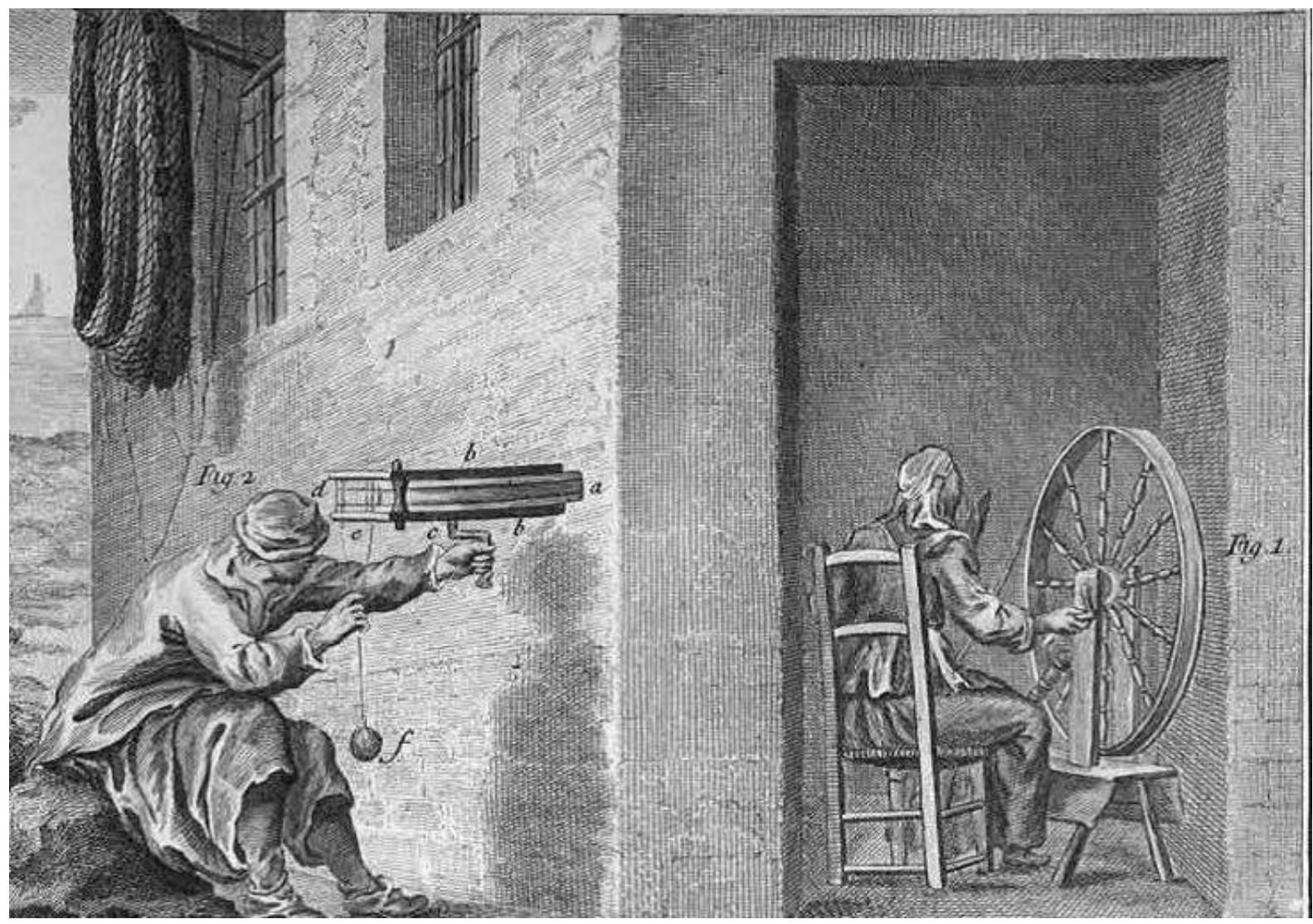

Les deux types de contradictions pointées par Duhamel du Monceau dans l'activité des pêcheurs

Mais cette activité est menacée économiquement par l'épuisement de la ressource vivante et par les coûts de production. Duhamel du Monceau repère alors deux types de contradictions: entre la réglementation royale sur la taille des mailles et le comportement physique du filet d'une part, entre la rentabilité de l'activité de pêche et les compétences des pêcheurs d'autre part. Si les contradictions relatives à la réglementation ne sont pas dépassées dans ce traité - bien que systématiquement soulevées dans un texte à plusieurs voix (celles de la réglementation, des groupes de pêcheurs et de l'auteur), les contradictions relatives à la rentabilité de l'activité seront, par contre, réglées par un exposé des techniques à maîtriser sur les filets de pêche.
Les contradictions entre les règlements et le comportement physique des filets

Le Traité des pêches de Duhamel du Monceau s'appuie sur les travaux de Le Masson du Parc. En 1726, une lettre patente royale (Louis XV, 23 avril 1726) dévolue à Le Masson du Parc l'inspection des installations de pêche, et plus particulièrement, « de se faire représenter les filets des pêcheurs, pour voir si les mailles sont conformes ». Ces ordonnances sont édictées car, dès le XVIII ${ }^{\mathrm{e}}$ siècle, des pratiques destructives ont été constatées (Busson, 1888). Le roi Louis XV écrit :

Il y a lieu d'espérer avec de pareilles dispositions, de faire cesser les abus qui se sont passés jusqu'à présent par rapport à la pêche, de conserver le frai du poisson et le poisson du premier âge.

Duhamel du Monceau est très sensible à la préservation et à l'exploitation de la ressource vivante dans le secteur de la pêche comme dans celui de la forêt 
(Dupont de Dinechin, 1999). Dès l'introduction générale du Traité des Pêches, Duhamel du Monceau présente la police des pêches ou "prud'hommes » composés de pêcheurs élus par la communauté:

Il a paru nécessaire de confier la police des pêches à ces prud'hommes, parce que les jugements sur le fait des pêches dépendent d'une infinité de combinaisons qui ne peuvent être connues que par ceux qui ont longtemps pratiqué toutes les différentes espèces de pêches. (\$ 25) l'eau de mer et lors du tannage. En d'autres termes, la règle lui apparaît inapplicable.

Duhamel du Monceau emploie un discours à plusieurs voix (Bronckart, 1996), les voix sociales de l'institution royale et des groupes de pêcheurs et la voix contradictoire de l'auteur introduisant un discours ( «logos») pour invalider la technique («tekhnê ») (Chevallard, 1992) imposée par la loi ou suggérée par les pêcheurs. Les éléments techniques et technologiques sont placés en vis-à-vis dans le tableau 1 ci-dessous:

Tableau 1. Mise en correspondance de deux « voix » : la réglementation et l'auteur

\begin{tabular}{|c|c|}
\hline Proposition technique et légale & Contre-argument technologique \\
\hline $\begin{array}{l}\text { (On a jugé qu'il était important de) «Fixer l'ouverture des mailles } \\
\text { que devait avoir chaque filet » }\end{array}$ & $\begin{array}{l}\text { «Il n'est pas aisé de mesurer exactement en pouces et en lignes } \\
\text { l'ouverture des mailles: aussi les pêcheurs ne suivent pas cette } \\
\text { méthode.» }\end{array}$ \\
\hline $\begin{array}{l}\text { «Ceux des ports du Ponants comptent combien il y a de nœuds }{ }^{22} \\
\text { au pied ou à la brasse \& ceux de Méditerranée combien il y a } \\
\text { d'ourdres }^{23} \text { au pan }{ }^{24} \text { ou à la brasse. " }\end{array}$ & $\begin{array}{l}\text { «Cette façon (...) est commode: mais elle n'est pas sûre; car en } \\
\text { supposant que la longueur soit telle qu'on l'exige au sortir des } \\
\text { mains de l'ouvrier [...] les fils se détendent, ils se détordent, } \\
\text { ils se crispent, ils augmentent de grosseur; ce qui diminue } \\
\text { considérablement l'ouverture des mailles. » }\end{array}$ \\
\hline $\begin{array}{l}\text { «Établir la grandeur des mailles sur le diamètre des moules qui } \\
\text { servent à les travailler." }\end{array}$ & $\begin{array}{l}\text { «La diminution des mailles devient plus ou moins considé- } \\
\text { rable, suivant la grosseur des fils qu'on a employé pour les } \\
\text { faire. » }\end{array}$ \\
\hline $\begin{array}{l}\text { (La loi prévoit qu') « il faudrait en même temps spécifier de quel fil } \\
\text { on se servirait." }\end{array}$ & $\begin{array}{l}\text { «Ce qui n'est pas aisé à vérifier, d'autant qu'il y a des fils qui se } \\
\text { gonflent à l'eau plus que d'autres. » }\end{array}$ \\
\hline $\begin{array}{l}\text { "On s'est donc beaucoup attaché dans les différents règlements } \\
\text { qu'on a faits relativement aux pêches, à fixer la grandeur des } \\
\text { mailles." }\end{array}$ & $\begin{array}{l}\text { "Quand on traîne le filet obliquement au courant, } \\
\text { les fils se rapprochent, les mailles s'allongent. " } \\
\text { "L'exacte dimension des mailles ne serait utile que pour les } \\
\text { filets qui seraient bien tendus et que l'on opposerait perpendicu- } \\
\text { lairement au courant. " "Ces circonstances sont assez rares. " }\end{array}$ \\
\hline
\end{tabular}

L'une des missions des prud'hommes est l'édiction de règles non écrites mais suivies "religieusement " pour la conservation de la ressource vivante. Les poissons les plus petits ne doivent pas être capturés pour pouvoir se reproduire:

Comme les prud'hommes de Marseille veillent également à ce qui peut rendre la pêche abondante \& à la conservation du petit poisson, ils jugèrent qu'il fallait empêcher que pour certaines pêches on se servit de trop petits ains ${ }^{20}$, afin qu'en en employant de plus gros, les petits poissons ne pussent pas s'y prendre. (§ 28)

Dans l'article second sur La fabrication des filets, Duhamel du Monceau introduit un alinea particulier intitulé: De la meilleure manière de constater la grandeur des mailles ${ }^{21}$. Il met en évidence les contradictions entre la règle de mesure des mailles confiée à la police des pêches et le comportement du filet dans
Ainsi, Duhamel du Monceau (et Le Masson du Parc auparavant) consigne les pratiques règlementées des pêcheurs pour les apprécier ensuite sur le seul plan technologique, sans référence à des théories savantes, conformément aux exposés du XVIII ${ }^{\mathrm{e}}$ siècle (Carnino, 2010). Les contre-arguments ci-dessus (partie droite du tableau) annoncent pourtant la physique des matériaux. Duhamel du Monceau prépare ainsi l'étude de l'activité de la pêche par différentes disciplines, comme la physique mais aussi l'économie, le droit. L'examen systématique des techniques permet d'évaluer leur efficacité et leur utilité pour le bien public (Picon, 2001). 


\section{Les contradictions entre la rentabilité économique et les compétences des pêcheurs}

Pour garantir la pérennité de l'activité, il s'agit, non seulement, de préserver la ressource vivante mais aussi, de produire des filets de qualité et de savoir les entretenir. Duhamel du Monceau fournit au lecteur des indicateurs de coût des filets et une quantification du travail:

Par exemple, les sennes ${ }^{25}$ pour le hareng sont les plus chères, non seulement à cause de leur hauteur, mais encore parce que les mailles sont fort serrées \& en grand nombre 26 ; ce qui fait qu'un habile laceur ne peut en faire par jour que huit à neuf aunes. Au contraire, un bon ouvrier peut faire douze à quinze aunes de manets ${ }^{27}$ qui servent pour la pêche du maquereau, dont cependant les pièces ont quarante-deux à quarantequatre mailles de chute. ( $\$ 14)$

Pour justifier l'exposé, dans le Traité, d'une technique donnée, Duhamel du Monceau affirme que celle-ci n'est pas maîtrisée par tous les pêcheurs. L'article 12 sur le raccommodage des filets est introduit ainsi :

Bien des gens qui savent faire des filets, ignorent la manière de les raccommoder. Cependant, comme nous l'avons dit, il est plus important aux pêcheurs de raccommoder, radouber ou ramender par eux-mêmes leurs filets, que de savoir en faire de neufs, puisque l'entretien des filets en prolonge la durée de plus de moitié. Un filet qui a quelques mailles de rompues aura bientôt un grand trou, si on ne le raccommode pas au plus tôt. (§ 164)

Ainsi, ce sont solidairement la préservation de la ressource, le rendement économique et la maîtrise des techniques qui permettent de pérenniser l'activité de pêche et de maintenir ainsi les populations utiles à la marine de guerre sur le littoral. Le Traité fait entrer ces techniques dans un monde « objectif » c'est-à-dire des connaissances fondées sur les critères de vérité et d'efficacité (Bronckart, 1996 ; Habermas, 1987). Pour cela, Duhamel du Monceau doit transposer par écrit les gestes des pêcheurs préalablement recueillis par enquête ou observation ${ }^{28}$. Ce sont les procédés didactiques utilisés à cette fin qui seront présentés dans la partie suivante de cet article.

\section{L'AMBITION DIDACTIQUE: L'ANALYSE DES PROCÉDÉS DE TRANSMISSION ÉCRITE DES TECHNIQUES DE PÊCHE}

Pour accroître les compétences de ses lecteurs, Duhamel du Monceau doit mettre en place différents procédés de « compétentialisation » (Greimas $\&$ Fontanille, 1984, p. 124) depuis les articulations entre les différents segments du texte jusqu'à des procédés plus profonds tels le choix des pronoms et des voix qui s'expriment (Bronckart, 1996) ou encore différents rapports entre le texte et les figures de la fin de l'ouvrage. Cette partie de l'article précisera ces procédés et leurs effets didactiques.

\section{Une organisation feuilletée du texte}

C'est la première fois dans l'histoire de la pêche que la modalité écrite est utilisée pour diffuser les techniques des pêcheurs. Comme tout texte écrit, le Traité des Pêches doit apporter de nouvelles composantes définies ainsi par Reuter (2006):

Écrire, c'est tout à la fois fixer/inscrire, abstraire, et rendre visible. Labstraction est à comprendre comme décontextualisation des situations, interactions et relations qui leur sont liées, du monde, du flux langagier, de la personne et séparation des unités, des acteurs (ceux qui composent, ceux qui exécutent, les producteurs, les récepteurs des messages). Le fait de rendre visible se comprend comme décrochage des contraintes temporelles liées à l'oralité et comme adjonction des dimensions spatiales et visuelles... À ce niveau, l'écriture génère un objet langagier et instaure une distance spécifique au regard des fonctionnements de l'oralité dans les cultures de l'oralité. (p. 140)

Bien que le Traité des Pêches possède les propriétés d'abstraction et de visibilité par exposé et illustration des techniques, celles-ci ne peuvent suffire. Le texte s'apparente, en effet, aux textes universitaires magistraux décrits par Bouacha (1981). Dans un cours universitaire, le discours linéaire de l'enseignant, bien que décontextualisé c'est-à-dire sans références à l'enseignant, à l'auditeur ou à la situation d'écoute - sans doute, parce qu'il est produit en modalité écrite avant d'être dit - est périodiquement rompu par des « décrochements » marqués par le terme "alors » à l'oral et par la prise en compte des auditeurs. 
Bronckart (1996) désigne ce type particulier d'articulation comme « interactif-théorique » car il permet d'articuler deux « attitudes de locution » différentes:

Il (ce type mixte) découle de la double contrainte qui s'exerce sur leur auteur; celui-ci doit présenter les informations qui constituent à ses yeux des vérités autonomes et qui s'inscrivent en conséquence dans les coordonnées d'un monde théorique; et il doit simultanément tenir compte des récepteurs, les solliciter, et s'inscrire dans les coordonnées d'un monde interactif (Bronckart, 1996, p. 259).

Document 2. Alternance des segments et différents types de décrochements

\section{A R T I C L E Q U A T R I E M E. \\ Maniere de trayailler les filets.}

86. IL ne fuffit pas de favoir faire les nœuds : cette connaiffance ferait inutile, fi l'on ignorait comment on forme les mailles. Nous avons déjà dit qu'il $y$ en a de deux fortes; celles qui forment des lofanges, \& celles qui font quarrées. Nous allons expliquer féparément la maniere de les faire.

Segment 1 - Décrochement: Duhamel du Monceau revient sur ce qui a été exposé dans la partie précédente et introduit le nouvel objet d'étude.

\section{Maniere de travailler les filets dont les mailles font en lofange.}

87. IL faut commencer par faire ce qu'on nomme la levure, qui eft compofée d'un nombre de demi-mailles qui forment la tête du filet. A cet égard, la pratique des mailleurs n'eft pas uniforme.

88. LFs uns ayant fait une anfe de ficelle G ( $p l$. I, fig. Io), la paffent dans un crochet $F, \& y$ attachent par un nœud fimple le fil dont ils doivent faire le filet; puis plaçant le moule fous le nœud qui elt au-bas de l'anfe $G$, ils font la maille $\mathrm{H}$; ils retirent le moule de cette maille, le pofent deffous, \& font la maille I, dont les branches font d'inégale longueur, ainfi qne toutes les autres, jufqu'au bout de la levure; ils tirent enfuite le moule de la maille I, pour le placer deffous, \& faire la maille $\mathrm{K}$. Ils font de mème \& fucceffivement les mailles LMNO, \&c. Comme le mailleur doit tirer fortement fur les mailles. qu'il a faites, elles font fermées \& les fils font rapprochés tout près les uns des autres; cependant nous les avons repréfentés un peu écartés, pour qu'on pût fe former une idée de la forme que les mailles prennent : d'ailleurs, on ne fait: ufage de cette levure, qu'en ouvrant les mailles, \& paffant une ficelle dans celles qui font cottées HKMO; ce qui eft repréfenté par la ligne ponctuée P Q. Mais comme la levure qu'on vient de faire, fe racoourcit à peu près de moitié, lorfqu'on ouvre les mailles, il faut la faire une fois plus longue que: ne doit être la tête du filet. Si cette tète doit avoir : quatre pieds de longueur, il faut que la longueur de la levure foit de huit pieds.

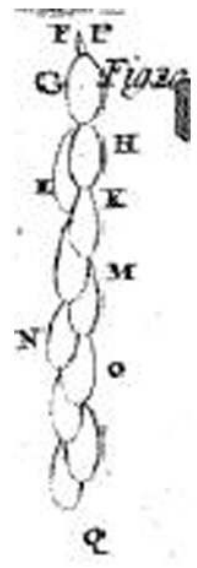

Segment 2 - Discours technique: Duhamel du Monceau décrit les techniques de création des mailles des paragraphes 87 à 102. Son discours fait référence à une figure d’une planche située en fin d'ouvrage.

Duhamel du Monceau expose ensuite de façon générale le maillage des filets (partie non reproduite). 
97. MaIs il eft bon de faire remarquer qu'on fait toujours les filets de mailles, de gauche à droite. Ainfi, quand une rangée eft faite dans toute la largeur du filet, on doit le retourner pour revenir fur fes pas, \& faire la feconde rangée, toujours de gauche à droite, \& les fuivantes de meme, jufqu’à ce que le filet foit achevé.

Segment 3 - Décrochement: Duhamel du Monceau met en exergue un aspect de la technique.

Duhamel du Monceau reprend la technique en insistant sur le retournement du filet à chaque rang (partie non reproduite).

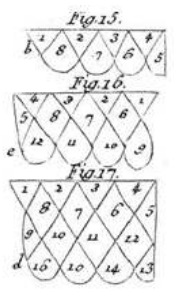

IOI. LA frgure I 6 repréfente le filet retourné pour faire la maille 9 , qui a fes deux branches inégales, enfuite les mailles IO, I I.\& I 2. $c$ eft le fil qui refte pour faire la rangée fuivante, quand on aura retourné le filet; \& l'on peut remarquer que les mailles $5,6,7 \& 8$, qui étaient arrondies par en-bas à la figure is, font anguleufes dans la figure $16, \&$ qu'elles forment des lofanges régulieres. Quand on a retourné le filet, comme on le voit dans la figure 17,

Segment 4 - Décrochement: Duhamel du Monceau requiert l'attention du lecteur sur un ensemble de figures.

102. Nous ne pourfuivrons pas plus long-tems le flet: ce que nous venons de dire, fera comprendre ou font les attaches des différentes mailles; comment les mailles qui font arrondies au fortir du moule, deviennent lofanges; \& comment, à caufe des attaches, il y a au bord du filet des mailles longues, \& des demi-mailles qui forment une efpece de bordure.

Segment 5 - Décrochement: Duhamel du Monceau clôt le discours.

Ainsi, le texte du Traité des Pêches présente une organisation "feuilletée " répartie entre des segments de discours autonome c'est-à-dire sans interpellation du lecteur et des décrochements de différents types. Par exemple, dans l'article 3 de la section sur les filets de pêche, Duhamel du Monceau a présenté la manière de faire les nœuds. Il aborde, au début de l'article 4, la manière de " travailler les filets » c'est-à-dire de fabriquer les mailles. Les segments de discours autonome sont placés à gauche et les segments de décrochement sont placés à droite dans la présentation ci-dessus de ce feuilletage:

La structure feuilletée du texte conduit à distinguer une approche macroscopique de celui-ci de l'analyse microscopique des différents types de segments (segments autonomes, segments de décrochement). Par cette structure feuilletée et par des procédés précis au sein de chaque segment, le texte de Duhamel du Monceau met en relation l'auteur, le lecteur et le savoir. Aussi, dans la suite de l'article, la description de cette dynamique à deux niveaux emploiera les trois catégories d'élaboration du savoir définies par Sensevy (Sensevy, 2001, 2007, 2011; Sensevy, Mercier, \& Schubauer-Leoni, 2000) : le triplet mésogenèse, topogenèse et chronogenèse:

- La mésogenèse ${ }^{29}$ concerne les moyens de création et de redéfinition du contenu de savoir, de telle façon qu'il acquière une signification commune pour le lecteur et pour l'auteur. - La topogenèse ${ }^{30}$ permet de décrire la répartition des responsabilités entre l'auteur et le lecteur.

- La chronogenèse $e^{31}$ correspond aux évolutions du contenu par introduction d'un élément nouveau dans le milieu. Lauteur génère, en faisant avancer le temps didactique, un 
effet de chronogenèse, c'est-à-dire le parcours d'« une séquence, [d'] une suite orientée d'objets de savoir » (Sensevy, 2001, p. 209).

\section{Les effets et les procédés des décrochements}

Les décrochements ont pour effet général d'établir une relation didactique avec le lecteur. Dans cette partie, nous nous proposons de décrire cette relation en utilisant les catégories d'effets distingués par Sensevy, Mercier et Schubauer-Leoni.

\section{Des effets topogénétiques}

Dans les segments proprement techniques (tel le segment 2), le discours est abstrait de toute interaction, au risque d'une passivité du lecteur(Go \& Sensévy, 2000). L'auteur introduit donc des décrochements pour interpeller son lecteur et lui exprimer son intention (ex : segment 1), le lecteur devant, quant à lui, suivre la progression du texte et acquérir les techniques exposées. Duhamel du Monceau distingue ainsi les responsabilités et " les lieux du professeur et de l'élève » (Sensevy et al., 2000, p. 266) réalisant ainsi un effet de « topogenèse ».

\section{Des effets chronogénétiques}

Au-delà d'un effet topogénétique, certains décrochements (par exemple, les segments 1 et 5) permettent de récapituler, d'anticiper des liens entre différentes parties du texte.

Les procédés linguistiques utilisés dans ces décrochements à effet chronogénétique reposent sur l'emploi de pronoms et de tournures passives. Duhamel du Monceau s'adresse directement à son lecteur en utilisant surtout le pronom "nous ", désignant l'auteur ou des formes à tournures passives comme "il est bon». Ces emplois constituent des marques importantes de didacticité (Moirand, 1993) c'est-à-dire de communication d'une intention de rendre le lecteur plus compétent. En voici quatre exemples supplémentaires:

(6) Il y a bien des sortes de filets dans les trois genres dont nous venons de donner une idée; \& nous comptons en parler fort en détail, lorsque nous traiterons des pêches où l'on en fait usage. Mais il faut présentement passer à la fabrique des filets. (§ 21)

(7) Comme nous serons obligés d'employer quelques termes qui sont propres à l'art qui nous occupe, il est bon de commencer par les définir. (§ 40)

(8) Nous aurions bien d'autres choses à dire sur la proportion qu'il faut observer, suivant différentes circonstances, entre le lest et les flotteurs. Mais il sera mieux de n'en parler que quand l'occasion s'en présentera, relativement aux différentes pêches. (§ 199)

(9) Nous choisissons, pour expliquer comment on fait des accrues, un filet à mailles carrées (pl. I, fig. 19), parce que la démonstration en sera plus sensible. (§ 114)

On le voit, Duhamel du Monceau ne se contente pas d'imposer l'ordre d'exposé des objets techniques, il justifie ses choix auprès du lecteur: « nous comptons en parler lorsque...» (6), « il est bon de commencer... » (7), « il sera mieux de n'en parler... » (8), « parce que ... plus sensible...» (9). Les décrochements ont donc non seulement une fonction informative, transactionnelle mais permettent également d'établir une relation sociale avec le lecteur, cette seconde fonction étant fondée sur le « principe de politesse » (Alberdi Urquizu, 2009) qui atténue l'autorité et permet d'entrer civilement en contact. La politesse des tournures passives consiste à justifier la dissymétrie de pouvoir par l'affirmation d'un choix soucieux de l'intérêt du lecteur. Ainsi, l'effet chronogénétique est accompagné d'un effet topogénétique fondé sur une relation de politesse.

\section{Des effets mésogénétiques}

Enfin, certains décrochements permettent d'attirer l'attention du lecteur sur un aspect de la technique (segment 3 ) ou sur les propriétés des figures associées au texte (segment 4). Ils mettent en avant, pour le lecteur, différents objets procéduraux et graphiques dans le « milieu » et organisent les conditions de compréhension et d'apprentissage des techniques, c'est-à-dire la mésogenèse (Sensevy, 2001 ; Sensevy et al., 2000). Nous allons montrer ci-dessous que les effets obtenus sont à la fois topogénétiques et mésogénétiques 
En effet, dans les décrochements attirant l'attention du lecteur sur un point technique (ex: segment 3 ), le pronom personnel «nous » est employé par l'auteur pour définir son projet et faire adhérer le lecteur à celui-ci tandis que le pronom indéfini « on » permet à Duhamel du Monceau de "se décharger de la responsabilité de son énoncé " (Le Bel, 1991, p. 109) en laissant le lecteur s'impliquer dans la compréhension des techniques et dans l'utilisation de celles-ci selon les règles de l'art. En voici quelques exemples:

(10) Mais après ce que nous avons dit, on doit concevoir que quand on aura fait en dessous un autre rang de mailles, ces premières deviendront anguleuses... (§ 171)

(11) Il est clair qu'en continuant de travailler les autres rangs de mailles, comme nous venons de l'expliquer, on fera toute l'étendue du filet en mailles losangées. (§. 174)

(12) On peut maintenant concevoir comment, au moyen des accrues, on peut élargir un filet tant qu'on veut; car on peut former plusieurs accrues, comme C (référence à une figure), dans une file de mailles, \& augmenter le nombre des mailles proportionnellement au nombre des accrues. (§ 178).

Dans cette catégorie de décrochements, l'emploi différencié des pronoms établit une différenciation entre:

- Le couple " énonciateur-lecteur» par l'emploi $\mathrm{du}$ "nous » et des verbes " dire», " expliquer» pour l'énonciateur et du «on»associé au verbe de réflexion « concevoir » pour le lecteur;

- Le couple « lecteur-bon praticien de la pêche » par l'emploi du « on » associé au verbe « concevoir» pour le lecteur et le « on» associé aux verbes d'action « faire », « élargir », «former», « augmenter» pour le bon praticien de la pêche.

Le premier effet de cette opposition entre ces deux couples est topogénétique. Duhamel du Monceau met en mots une technique de pêche recueillie auprès des pêcheurs: son exposé de la technique lui apparaissant suffisant (« nous venons de l'expliquer » (11)), il invite le lecteur à anticiper et à généraliser («on doit concevoir» (10)) et lui indique que sa réflexion lui permet d'agir comme un mailleur (« on peut élargir »(12)). Il s'agit, dans les termes de la théorie des situations didactiques, d'une dévolution c'est-à-dire de « faire en sorte qu'il (ici, le lecteur) se sente responsable de l'obtention du résultat proposé » (Brousseau, 2003).

Le second effet de cette opposition est mesogénétique. Duhamel du Monceau clôt le segment technique en indiquant que la connaissance exposée dans son Traité des Pêches est suffisante pour agir comme un bon mailleur. Les décrochements discursifs rappellent ainsi l'authenticité des techniques exposées et l'intérêt de les utiliser. Cet effet mésogénétique est d'autant plus important que les lecteurs du Traité des Pêches pourraient être, idéalement, les pêcheurs eux-mêmes et que ce sont alors leurs propres gestes de pêche qui seraient confrontés aux techniques exposées.

Quant aux décrochements faisant porter l'attention sur les figures associées aux textes (segment 4), ils reposent, eux-aussi, sur l'emploi du pronom « nous » qui permet à l'auteur d'expliciter ses choix figuratifs tandis que le pronom «on » se réfère à toute personne examinant les figures. Les verbes « voir », « concevoir »-utilisés ici comme « se faire une idée » et « se représenter »-invitent les lecteurs à considérer le geste comme un objet conscient et à en construire une image mentale globale.

Enfin, sur un plan cette fois macroscopique, l'alternance entre les deux types de discours interactif et théorique a un effet de structuration des connaissances. Dans le cas des décrochements à effet mesogénétique (segments 3 et 4), l'alternance permet de passer d'une activité de lecture linéaire à une attention sélective à un aspect technique ou graphique. Dans le cas des décrochements à effet chronogénétique (segments 1 et 5), l'alternance entre les discours met en relation le savoir technique présenté à un moment donné par l'auteur et les savoirs passés et à venir. Si ce n'était pas le cas, comme le précise Bouacha pour les cours universitaires, l'apprentissage ne serait que l'adjonction d'un savoir nouveau à un savoir acquis:

C'est cet équilibre entre un savoir narrativisé dans un avant et un après et un savoir construit dans le maintenant du cours que réalise le cours universitaire. Transposé sur le plan du discours, cet équilibre se maintient entre un discours déjà dit (et qui reste à dire) et un discours en train de se dire; d'où des décrochements discursifs constants [...]. (Bouacha, 1981, p. 48). 
Les procédés et les effets des segments d'exposé technique

Le Traité des Pêches a pour ambition l'inscription conforme et exhaustive (propriété fixer/inscrire) des techniques de maillage recueillies sur le littoral. En premier lieu, le contenu technique du texte est fondé sur le principe mésogénétique que celui qui sait construire le filet en comprend la structure maillée et peut, dès lors, entreprendre de le réparer. Aussi, le contenu est ordonné chronogénétiquement depuis la création du filet à sa réparation et, enfin, à son entretien. En deuxième lieu, le contenu relatif au ramendage (réparation) du filet ne rend compte que des bonnes pratiques qui assurent à terre la pérennité du matériel et non des réparations de fortune par faufilage qui pourraient être effectuées en mer. Enfin, le texte est caractérisé par un effacement de l'auteur, du lecteur, et des mailleurs. Lorsque les ramendeurs et mailleurs sont évoqués, c'est pour présenter les variations observées de leurs techniques et les présenter une à une sans les hiérarchiser. Observons, par exemple, l'amorce du texte sur le maillage des filets dont les mailles sont en losange (article quatrième): « Il faut commencer par faire ce qu'on nomme la levure, qui est composée d'un nombre de demimailles qui forment la tête du filet. À cet égard, la pratique des mailleurs n'est pas uniforme. » (§ 87). Duhamel du Monceau expose les différentes techniques puis conclut: "C'est ainsi que les mailleurs ont coutume de travailler. » (§ 94)

- C'est le pronom impersonnel « on» qui est surtout employé. La personne amenée à accomplir le geste technique peut alors être n'importe qui, le lecteur comme un pêcheur, ce procédé annonçant ainsi une division possible du travail.

- Les injonctions telles que « il faut», « on doit $»$ sont peu fréquentes et sont surtout utilisées pour introduire un passage technologique de justification de la technique. Ainsi, à propos de la «levure », Duhamel du Monceau explique que:

[...] comme la levure qu'on vient de faire, se raccourcit à peu près de moitié, lorsqu'on ouvre les mailles, il faut la faire une fois plus longue que ne doit être la tête du filet. Si cette tête doit avoir quatre pieds de longueur, il faut que la longueur de la levure soit de huit pieds. (§ 88).
On le voit, les procédés utilisés dans les segments techniques conduisent à un effacement de l'auteur, des ramendeurs et du lecteur, c'est-à-dire de tout « topos» du savoir. Ainsi, le texte n'a pas de contenu incitatif explicite et il est acquis que le lecteur souhaite accomplir correctement le geste technique. Pour exposer le contenu technique, Duhamel du Monceau emploie des procédés d'ostension, c'est-àdire de désignation des gestes devant être nécessairement accomplis (Brousseau, 2003). Duhamel du Monceau propose, de ce fait, un « contrat implicite » (Manno, 2009) au lecteur comme pour les recettes de cuisine (Greimas, 1983): si vous faites comme il est indiqué, vous obtiendrez certainement le produit escompté. Plus le producteur du texte et le destinataire sont effacés, plus le contenu technique fait autorité. Dès lors, le Traité des Pêches transforme les techniques de pêche en œuvres (Meyerson, 1948/1995) c'est-à-dire en objets indépendants des pêcheurs qui ont font habituellement usage.

\section{Les rapports entre les figures et le texte}

Les discours techniques de Duhamel du Monceau portent sur la construction des filets, leur réparation et leur entretien. Comme il est d'usage dans les ouvrages encyclopédiques de cette époque, le texte se réfère à des figures regroupées dans des planches en fin d'ouvrage ou dans un ouvrage séparé, selon les éditions. Les figures sont numérotées et, dans la seconde édition, organisées sur la planche selon l'agencement spatial le moins coûteux. Dans les premiers articles de la section relative aux filets, les figures représentent des instruments des pêcheurs. Dans les articles suivants, les figures illustrent l'accomplissement du geste technique.

Le fait de « rendre visible » est une autre propriété utilisée par Duhamel du Monceau. En effet, en adjoignant des figures, l'auteur réalise un va-et-vient entre le texte et les figures et amorce une tabularité de l'ouvrage (Florea, 2009). Dès lors, l'auteur doit former son lecteur à un langage graphique et pour ce faire, une autre catégorie spécifique de décrochements est introduite dans le texte.

Nous considèrerons essentiellement trois extraits du texte, correspondant aux techniques de maillage encore importantes de nos jours ${ }^{32}$ :

- (extrait 1 ) « de la manière de faire les différents nouds qui joignent les fils les uns avec 
les autres » (article 3, § 66 à § 85) associé à la planche II de la section II;

- (extrait 2) « de la manière de travailler les filets dont les mailles sont en losange » (dans l'article 4, §87 à § 103) associé à la planche I de la section II;

- (extrait 3) « Raccommodage des filets » (article $12, \S 164$ à § 185), associé à la planche III de la section II.

Lorsqu'il amorce la rédaction du Traité des Pêches, Duhamel du Monceau dispose déjà des figures composées au début du XVIII ${ }^{\mathrm{e}}$ siècle par les dessinateurs employées par de la Marre. C'est donc a posteriori que Duhamel du Monceau établit trois types de rapports entre les figures et le texte (Apotheloz, 1998) au service de la mesogenèse des techniques.

\section{Lillustration: la figure illustre le texte}

L'illustration est le premier type de rapport. Dans ce cas, le texte décrit une suite d'actions et fait référence à des figures et à des objets précis de la planche. Tout objet (doigts, fils, mailles...) est désigné par une lettre ou un chiffre et l'ordre des actions ou des objets évoqués est traduit par l'ordre alphabétique ou l'ordre numérique. Par ces procédés de codage, Duhamel du Monceau établit des relations d'homomorphisme (Vergnaud, 1994) c'est-à-dire de correspondance entre le texte et la représentation figurative, comme dans le segment suivant:

Document 3. L'illustration du texte par la figure.
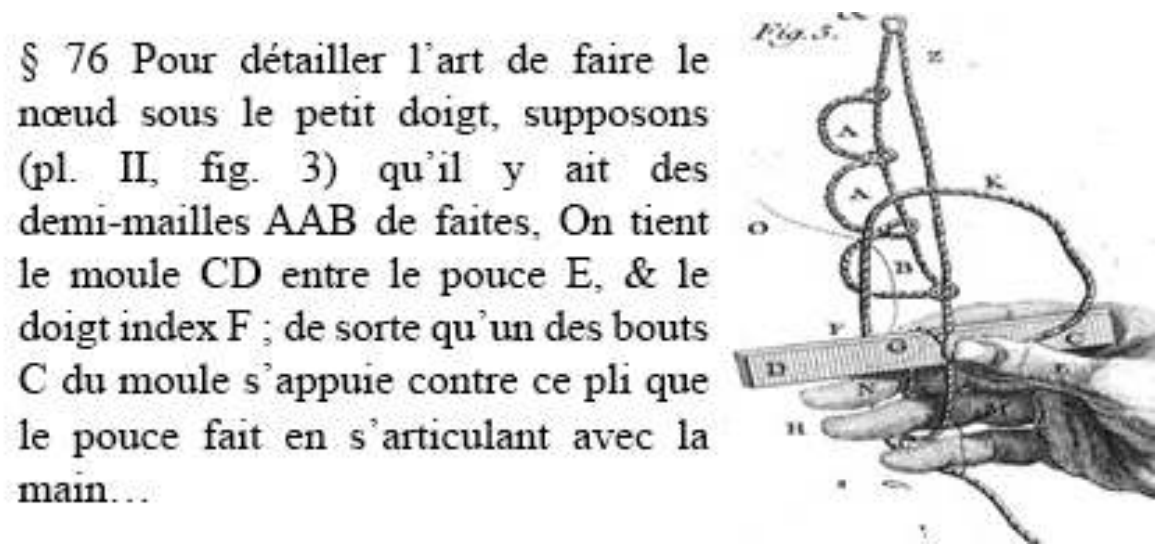

La description: le texte décrit la figure

La description est le deuxième type de rapports. Elle est observée dans la troisième catégorie de décrochements décrite précédemment (segment 4). L'auteur incite les lecteurs à examiner des figures particulières afin qu'ils acquièrent un langage graphique de description des gestes et du filet de pêche. Ce langage permet, en particulier, de s'affranchir de ce que l'on voit et de ce l'on fait à un moment donné. Ainsi. Duhamel du Monceau fait remarquer à son lecteur que l'image ne représente qu'un état transitoire: 
Document 4. La description de la figure par le texte.

96. Ces demi-mailles, qui font faites fur un moule, paraifent arrondies par en-lias; mais on verra bientôt que quand on fera les mailles du premier rang, femblables à I $\mathbf{3}, \mathrm{I}_{4}, \mathrm{i} \mathbf{5}, \& \mathrm{c}$. qui s'attachent au milieu des demimailles $4,5,6$, ces demi-mailles qui étaient arrondies, comme celles $\mathbf{I}, \mathbf{2}$ $\& 3$, feront devenues triangulaires, ainfi que toutes les fuivantes depuis: 4 jufqu'à I2. De même les mailles $\mathrm{r}_{3}, \mathrm{I}_{4}, \mathrm{I} 5$, qui font arrondies par enbas, deviendront anguleufes, \& formeront des lofanges femblables à 16 ,

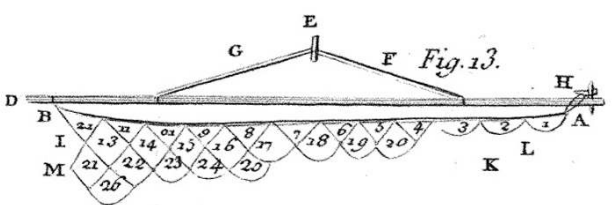

Ou encore que des pointillés permettent de figurer " la route que doit tenir le fil pour achever le noud », le caché devenant visible et le fugace permanent sur la figure 3 déjà reproduite ci-dessus:

Document 5. L'introduction du codage des figures.

\section{La fgure 4 eft deftinée à faire concevoir le refte de ce nœud. Nous avons cependant tracé fur la figure 3 , par une ligne ponctuée, la route que le fil doit tenir pour achever le nœud. Comme nous avons omis exprès de repré- fenter l'aiguille dans cette figure 3 , on apperçoit mieux les différens contours}

Au-delà des conventions générales de notation des figures, le rapport de description permet de nommer définir les concepts spécifiques des instruments du pêcheur. En effet, en introduisant peu à peu une terminologie, Duhamel du Monceau permet à ses lecteurs de nommer les objets et de les mettre en relation dans la structure matérielle du filet. Le discours appelle une éducation du regard par les concepts de nœud, de jambe, d'oblique, de branche... L'exemple ci-dessous concerne le ramendage: la connaissance des composantes du filet est décisive dans cette activité car réparer, c'est rétablir une structure.

Document 6. L'éducation du regard: les concepts de jambe et de nœud.

177. VoILA en gros la marche qu'on doit fuivre pour r'habiller les filets. Mais ces idées générales ne fuffifent pas; nous devons entrer dans queiques. détails fur la pratique ufitée dans l'art de faire les nocuds, tant pour les mailles. que pour les jambes: c'eft à quoi nous allons effayer de fatisfaire. On appelle jambe un fil qui, étant feul \& dans une direction oblique, fuffit pour établir la liaifon que doivent avoir réciproquement deux nouds quine font pas fur. une méme ligne, tel que E D \& I K ( f g . 4).

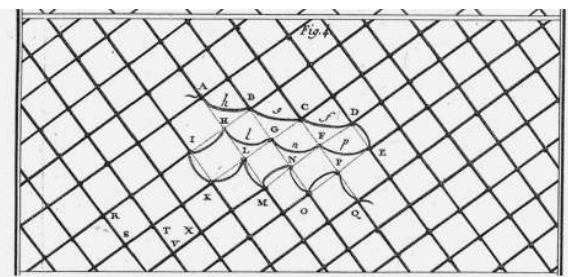




\section{L'éclatement du geste dans différentes figures}

Enfin, l'examen de la section sur les filets met en évidence la structuration d'un ensemble de figures par le discours. En effet, chaque technique est éclatée dans différentes figures (Lavoisy, 2000, 2004). Certaines figures permettent alors d'isoler une étape particulière de la technique, d'autres permettent de montrer le geste d'ensemble, d'autres encore de montrer ce qui est caché par la main ou l'aiguille... Ainsi, comme nous l'illustrons ci-dessous pour le nœud sous le petit doigt, si la lecture locale d'une étape d'une technique donnée met en avant un rapport d'illustration du texte par la figure (\$ 76) ou un rapport de description (§ 79), la lecture d'ensemble de la technique révèle, quant à elle, une mise en relation par le discours des différentes figures:

\section{CONCLUSION}

Le Traité des Pêches, et plus particulièrement la section sur les filets de pêche, nous a permis de montrer l'imbrication du cadre politique et philosophique, du cadre documentaire et du cadre didactique dans une œuvre encyclopédique du XVIII ${ }^{\mathrm{e}}$ siècle. C'est la seule fois dans l'histoire de l'enseignement technique de la pêche que l'on parle ainsi de tout à tout le monde: des techniques de pêche à des nonpêcheurs et du contexte social, légal et économique de la pêche à des pêcheurs. Or, on sait que les techniques de pêche d'aujourd'hui, comme celles d'hier, sont déterminées par des enjeux légaux, environnementaux et économiques qu'il faut articuler entre eux. De plus, bien avant que des théories savantes ne soient développées sur l'enseignement et l'apprentis-

Document 7. L'éclatement du geste dans des figures différentes et sa recomposition par le texte.

\$76 Pour détailler l'art de faire le nœud sous le petit doigt, supposons (pl. II, fig. 3) qu'il y ait des demi-mailles $\mathrm{AAB}$ de faites, On tient le moule $\mathrm{CD}$ entre le pouce $\mathrm{E}$, \& le doigt index $\mathrm{F}$; de sorte qu'un des bouts $\mathrm{C}$ du moule s'appuie contre ce pli que le pouce fait en s'articulant avec la main...

$\S 79$ La figure 4 est destinée à faire comprendre le reste de ce nœud. Nous avons cependant tracé sur la figure 3, par une ligne ponctuée, la route que le fil doit tenir pour achever le nœud.

$\S 80$ Pour rendre encore plus clair ce que nous venons de dire, nous distinguons en trois opérations ce qui regarde le nœud sous le petit doigt.

§81 À la première qui est représentée par la figure 3 , on passe le fil entre le moule \& l'extrémité du pouce en $\mathrm{G}$.

$\S 82$, Pour la seconde, qui est désignée dans la même figure par une ligne ponctuée ... On conçoit que l'aiguille qui est représentée dans la figure 4, est nécessaire pour faire passer le fil par la route que nous venons d'indiquer, \& qui est désignée par les mêmes lettres dans les figures 3 et 4 .

$\S 83 \mathrm{La}$ troisième et dernière opération est représentée par la figure 5, qui fait apercevoir comment tous les doigts ayant été dégagés de l'anse MN (fig. 3, 4) le petit doigt qui demeure seul entouré du fil, s'élève avec lui peu à peu jusqu'au moule et ne se dégage que quand on est près de serrer le nœud. Alors, si on tire fortement le bout O du fil (fig. 3), qu'on doit toujours supposer tenir l'aiguille, le nœud est fini.

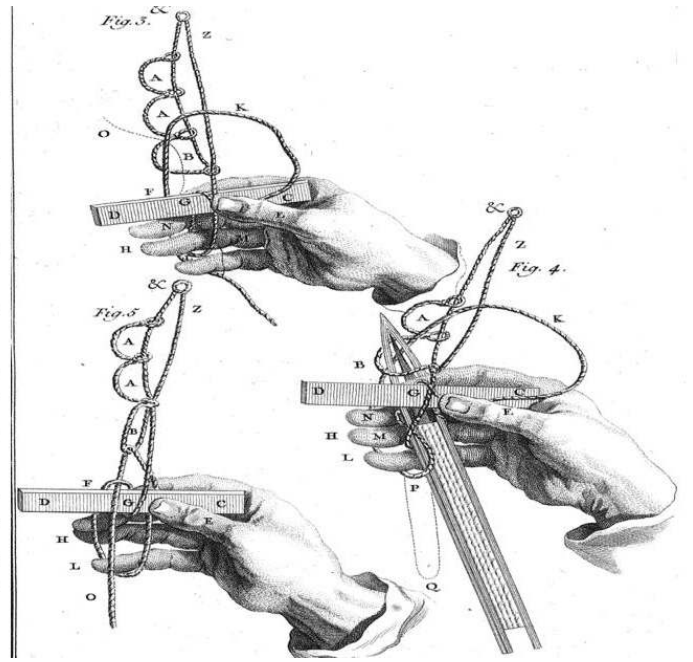

Les trois rapports que nous venons de distinguer préparent le lecteur à une utilisation autonome des planches de figures, celles-ci n'étant pas a priori des guides de réalisation pouvant être utilisés séparément par les novices. Ainsi, l'apprentissage de la pêche par l'écrit requiert au XVIII ${ }^{\mathrm{e}}$ siècle un temps d'étude au cours duquel les lecteurs apprennent non seulement des techniques mais également un langage descriptif et graphique ${ }^{33}$. sage, ce traité met en œuvre un " génie didactique » (Mercier, Rouchier, \& Lemoyne, 2001, p. 235) dont nous avons décrit les procédés. Ceux-ci ont pour effets, malgré la linéarité du texte, d'établir une relation didactique et de mettre en place un langage graphique et des rapports entre texte et figures.

Deux siècles plus tard, des documents techniques rédigés en France par l'ISTPM et Ifremer, organismes d'État, diffuseront auprès des seuls pêcheurs les normes techniques et terminologiques relatives aux chaluts. Il ne s'agira plus de diffuser les techniques 
des pêcheurs et des ramendeurs mais celles des techniciens (Libert \& Maucorps, 1969), la division du travail étant désormais actée.

Quant à l'effort d'utilisation d'une modalité écrite pour l'enseignement des techniques relatives aux filets, il ne sera poursuivi que dans le seul manuel scolaire publié à ce jour, manuel conçu et diffusé au Québec ${ }^{34}$ (Boudreau \& Myre, 1998). Ainsi, cet article sur le Traité des Pêches contribue à fonder une didactique de la pêche car, bien qu'ancien, le Traité demeure encore aujourd'hui une référence presque unique. 


\section{NOTES}

1. Cette encyclopédie est accessible en ligne sur le site de l'Université de Chicago http://encyclopedie.uchicago.edu/ dans le cadre du projet « American and French Research on the Treasury of the French Language » (Université de Chicago, CNRS).

2. Dupont de Dinechin est l'auteur d'une importante biographie de Duhamel du Monceau.

3. Nous prendrons pour référence les deux premières éditions. La première édition ou "édition de Paris» (1769) : Traité général des Pesches et histoire des poissons qu'elles fournissent tant pour la subsistance des hommes que pour plusieurs usages qui ont rapport aux arts et au commerce. L'édition de Paris est disponible sur le site archive.org: http://archive.org/details/ TraitGnralDesPeschesEtHistoireDesPoissonsQuelles Fournissent (partie 1 du Traité) et http://archive.org/ details/p2TraitGnralDesPeschesEtHistoireDesPoissonsQuellesFournissent (partie 2 du Traité). Les 20 volumes de l'édition suivante de la Description des Arts et Métiers, l'édition de Neuchâtel (1771-1783) sont disponibles en ligne sur le site du Conservatoire Numérique des Arts et Métiers http://cnum.cnam.fr. Le Traité des Pêches correspond aux volumes $\mathrm{V}$ et $\mathrm{X}$ de la deuxième édition de la Description des Arts et Métiers. La deuxième édition introduit un découpage en paragraphes.

4. Cette édition a été réalisée par Connaissance et Mémoires Européennes, éditeur à Paris: http://www.connaissancememoires.fr/titre_fiche.php?id_titre $=11$

5. Nous remercions Gaétan Myre, consultant en techniques de pêches au Québec de nous avoir fait connaître l'œuvre de Duhamel du Monceau. Nous remercions également Yoland Plourde, Joël Gaborit, Jean Guénolé, enseignants de techniques de pêche au Québec et en France pour leur lecture attentive des passages du Traité des Pêches relatifs aux techniques de maillage et à la reproduction de ces techniques.

6. Le mot exactitude est utilisé par Duhamel du Monceau lui-même (Duhamel du Monceau, 1776, p. 25).

7. Le choix de cette section est justifié par son inscription dans un projet de recherche sur l'apprentissage des savoirs relatifs au chalut, et plus particulièrement des compétences de ramendage (réparation) des filets de pêche.

8. Nous emploierons « TP » pour désigner les citations du Traité des Pêches de la deuxième édition dans cet article.

9. Le mailleur est l'artisan qui construit le filet de pêche. Le mailleur peut être le pêcheur lui-même. Duhamel du Monceau distingue à dessein les deux fonctions pour insister sur les types de tâches accomplies et sur une possible division du travail.

10. «Enlarmer un filet, c'est le border d'une espèce de lisière de grandes mailles, faites de fil fort, ou pour forti- fier le filet ou pour former des anneaux comme ceux d'un rideau: c'est à peu près ce qu'on nomme en Provence chappe. " "Accrues: les accrues sont de fausses mailles, ou surnuméraires que l'on met au bord du filet quand on veut augmenter la largeur. Pour les diminuer, on joint ensemble deux mailles par un même nœud. » (Table des matières du Traité des pêches)

11. Le projet des Encyclopédistes et celui de l'Académie des Sciences furent concurrents et ils utilisèrent plusieurs sources communes (écrits et planches de figures).

12. Duhamel du Monceau ne présente pas cette partie préparatoire de son travail et de celui de Le Masson du Parc. On notera cependant que, dans le Discours préliminaire à l'Encyclopédie, Jean Le Rond d'Alembert témoigne de la difficulté de parvenir à la mise en mots des gestes par les professionnels: "On s'est adressé aux plus habiles de Paris et du royaume ; on s'est donné la peine d'aller dans leurs ateliers, de les interroger, d'écrire sous leur dictée, de développer leurs pensées, d'en tirer les termes propres à leurs professions, d'en dresser des tables, et les définir, de converser avec ceux de qui on avait obtenu des mémoires, et (précaution presqu'indispensable) de rectifier dans de longs et fréquents entretiens avec les uns, ce que d'autres avoient imparfaitement, obscurément, et quelquefois infidèlement expliqué. »

13. «Sa main nue quelque robuste, infatigable et souple qu'elle soit, ne peut suffire qu'à un petit nombre d'effets; elle n'achève de grandes choses qu'à l'aide des instruments et des règles, il en faut dire autant de l'entendement. » (Diderot, Article Art)

14. Hélène Vérin (2000) rapproche, par anticipation sur la création des Arts et Métiers et de Polytechnique, la démarche de Duhamel du Monceau de celle des Arts et Métiers et l'oppose à celle de la formation de l'École Polytechnique qui guide l'action de l'ingénieur par l'application des lois scientifiques.

15. Ces corps de maîtrise technique existeront plus tard. En témoignent la création, en France, de l'Institut Technique des Pêches Maritimes, intégré aujourd'hui à Ifremer ou encore la création d'un diplôme collégial de technicien des pêches au Québec.

16. Remarquons que le terme de division du travail est associé à l'œuvre d'Adam Smith, qui s'appuie d'ailleurs sur une description de l'art de l'épinglier (Tome VII de la Description des Arts de Métiers, Édition de Neuchâtel). Cette description établie par Réaumur est complétée par Duhamel du Monceau et Perronet. Duhamel du Monceau en rédige l'introduction: «Il n'y a personne qui ne soit étonné du bas prix des épingles ; mais la surprise augmentera sans doute quand on saura combien de diffé- 
rentes opérations, la plupart fort délicates, sont indispensablement nécessaires pour faire une bonne épingle. Nous allons parcourir en peu de mots ces opérations pour faire naître l'envie d'en connoître les détails ; cette énumération nous fournira autant d'articles qui feront la division de ce travail. » (p. 531) Selon Paucelle, l'expression « division du travail » signifierait ici le plan suivi dans le texte et non la « division du travail de l'épinglier ». La reprise de l'expression par Smith serait donc une erreur d'interprétation qui s'est avérée productive sur le plan scientifique. Il reste, cependant, que l'art de l'épinglier s'attache à décomposer les nombreuses étapes de production des épingles.

17. Duhamel du Monceau définit ainsi le terme de ralingue dans la table des matières: « on les attache au bord des filets avec des ganses pour les fortifier. ». Une ganse est un cordonnet, un lacet, ici un « ainard ».

18. Il est remarquable que la figure à droite, représentant la femme, ait disparu dans le réaménagement des planches réalisé pour la seconde édition (édition de Neuchâtel).

19. La première édition du Traité des Pêches date de 1769 , soit 20 ans avant la Révolution, l'ordre social établi n'est nullement remis en cause par l'auteur.

20. Ain, hain ou Haim: hameçon.

21. Les paragraphes 29 à 32 de la section sur les filets de pêche sembleront très actuels aux pêcheurs d'aujourd'hui soumis aux règlementations sur la taille des mailles de filets pour préserver la ressource vivante. Cette préoccupation a plus de trois siècles car Duhamel du Monceau cite l'ordonnance de 1685 qui «a fixé la grandeur des mailles pour toutes les espèces de filets $\&$ a ordonné qu'il serait déposé au greffe des amirautés, des échantillons de toutes les espèces (de filet) pour avoir sous les yeux un objet de comparaison." "

22. Nœuds: "Les fils des filets sont joints les uns aux autres par des nœuds. [...] Pour constater la grandeur des mailles d'un filet, on compte combien il y a de nœuds dans une longueur, comme par exemple un pied. »

23. Ourdre: «Terme provençal, qui signifie ce que dans les ports du Ponant on appelle nœud.»

24. Pan: «Mesure d'usage en Provence, qui a neuf pouces de longueur. »

25. Duhamel du Monceau utilise l'orthographe "saine" pour l'actuelle "senne", technique qui consiste à placer un filet en surface pour encercler le poisson.

26. Duhamel du Monceau introduit ici la note de bas de page suivante: "On les fait, pour la plupart, de grossière soie de Perse, parce qu'ils sont plus forts, \& qu'ils peuvent durer trois ans. Anderson, Mémoires sur l'Islande. »

27. Voici la définition du terme « manet » dans le glossaire en fin de Traité des Pêches: «Filet en nappe simple dont les mailles sont proportionnées à la grosseur des poissons qu'on se propose de prendre ; ainsi, elles sont plus serrées pour les sardines que pour les harengs, \& pour les harengs que pour les maquereaux. Ceux pour prendre les mulets ont des ailes encore plus larges ; car il faut que la tête du poisson entre dans la maille, \& qu'il soit retenu par les ouies."

28. Cf. note 12 .

29. Mesos: «milieu»

30. Topos: «lieu».

31. Chronos: «temps».

32. Les techniques relatives à la conservation des filets ne sont pas reprises dans cet article car elles concernent à l'époque de Duhamel du Monceau les filets en chanvre, matériau aujourd'hui abandonné au profit des matières synthétiques.

33. Nous avons demandé à des enseignants de techniques de pêche également anciens pêcheurs de reproduire quelques techniques du Traité des Pêches. Certaines techniques, telles que celle de la levure (qui permet d'amorcer le maillage du filet) ne sont plus utilisées de nos jours, les nappes de filet étant obtenues mécaniquement. Les passages correspondants sont plusieurs fois relus par ces experts tandis qu'ils ne se servent d'autres passages du texte que pour confirmer que la technique exposée est semblable à celle qu'ils emploient eux-mêmes. Les figures sont alors d'un usage autonome pour ces personnes, habituées à ce langage graphique.

34. Ce manuel fait l'objet d'un article en préparation par Gaétan Myre. 


\section{RÉFÉRENCES}

Alberdi Urquizu, C. (2009). Politesse, savoir-vivre: modernité d'un concept bien classique. Synergies Espagne (2), 117-128.

Allard, M. (1970). Henri-Louis Duhamel du Monceau et le ministère de la Marine. Ottawa Léméac.

Apotheloz, D. (1998). Eléments pour une logique de la description et du raisonnement spatiali. In Y. Reuter (Éd.), La description. Théories, recherches, formation, enseignement. (p. 15-31). Villeneuve d'Ascq Presses Universitaires du Septentrion.

Artz, F.-B. (1938). L'éducation technique en France au $\mathrm{XVIII}^{\mathrm{e}}$ siècle (1700-1789). Revue d'histoire moderne, 13(35), 361-407.

Bandura, A. (1977). Social learning theory. Englewood Cliffs, N. J : Prentice-Hall.

Bertrand, J.-É. (1771). Préface Descriptions des Arts et Métiers (Vol. I, p. I-XIII). Neuchâtel: J.-E. Bertrand.

Bouacha, A.-A. (1981). «Alors» dans le discours pédagogique: épiphénomène ou trace d'opérations discursives? Langue française (50), 39-52.

Boudreau, M., \& Myre, G. (1998). Le ramendage, la réparation des filets de pêche. Grande-Rivière (QC) : Cégep de la Gaspésie et des Îles.

Brière-Guenoun, F., Perez, S., \& Durey, A. (2007). Étude exploratoire des connaissances mécaniques mobilisées par les enseignants d'EPS- Les conceptions des conseillers pédagogiques. Science et Motricité, 2(61), 9-23.

Briggs, R. (1991). The Académie Royale des Sciences and the Pursuit of Utility. Past $E$ Present(131), 38-88.

Bronckart, J.-P. (1996). Activité langagière, textes et discours. Pour un interactionnisme socio-discursif. Lausanne: Delachaux et Niestlé.

Busson, R. (1888). Les établissements de pêche et le domaine public maritime - Aperçu historique. Paris: Librairie militaire de L. Beaudoin et Co.

Carnino, G. (2010). Les transformations de la technologie: du discours sur les techniques à la «techno-science ». La technique, 2010(4), 75-84.

Chartier, R. (1987). Lectures et lecteurs dans la France d'Ancien Régine Paris: Éditions du Seuil.

Chartier, R. (1992). L'ordre des livres- Lecteurs, auteurs, bibliothèques en Europe entre $\mathrm{XIV}^{\mathrm{e}}$ et $\mathrm{XVIII}^{\mathrm{e}}$ siècle. Aixen-Provence: Éditions ALINEA.

Chevallard, Y. (1985). La transposition didactique. Du savoir savant au savoir enseigné. Grenoble: La pensée sauvage.

Chevallard, Y. (1992). Concepts fondamentaux de la didactique: Perspectives apportées par une approche anthropologique. Recherches en Didactique des Mathématiques, 12(1), 73-112.

Delbos, G., \& Jorion, P. (1984). La Transmission des savoirs. Paris: Éd.. de la Maison des Sciences de l'homme.

Deschamps, G. (2009). Les filets maillants. Versailles: Éditions Quae.
Duhamel du Monceau, H.-L. (1776). Traité des Pêches et Histoire des Poissons (sections I, II et III)- Tome V de la Description des Arts et Métiers. Neuchâtel: J.-E. Bertrand.

Dupont de Dinechin, B. (1999). Duhamel du Monceau. Un savant exemplaire au siècle des Lumières. Paris: Connaissance et mémoires européennes.

Engeström, Y., \& Sannino, A. (2010). Studies of expansive learning: Foundations, findings and future challenges. Educational Research Review(5), 1-24.

Florea, M.-L. (2009). Tabularité: des textes aux corpus. Corpus(8), 177-196.

Garçon, A.-F. (2006). Vents et fours en paléométallurgie du fer, du minerai à l'objet (C. d. h. d. techniques, Trans.). Paris: Université Paris 1 Panthéon-Sorbonne.

Go, H., \& Sensévy, G. (2000). Qu'est-ce qu'un cours de philosophie Diotime-L'Agora(8), 4-5.

Habermas, J. (1987). Théorie de l'agir communicationnel. Paris: Fayard.

Habermas, J. (1997). L'Espace public: archéologie de la publicité comme dimension constitutive de la société bourgeoise. Paris: Payot.

Hilaire-Perez, L., \& Thébaut-Sorger, M. (2006). Les techniques dans l'espace public- Publicité des inventions et littérature d'usage au XVIII ${ }^{\mathrm{e}}$ siècle (France, Angleterre). Revue de synthèse, $5^{e}$ série(2), 393-428.

Jaoul, M., \& Pinault, M. (1982). La collection « Description des Arts et Métiers » : Étude des sources inédites de la Houghton Library Université Harvard. Ethnologie française, 12(4), 335-360.

Kergoat, D. (2000). Division sexuelle du travail et rapports sociaux de sexe. In F.-L. Helena Hirata, Hélène Le Doaré et Senotier, D. (Éd.), Dictionnaire critique du féminisme (p. 34-44). Paris: Presses Universitaires de France.

Lavoisy, O. (2000). La Matière et l'Action-Le graphisme technique comme instrument de la coordination industrielle dans le domaine de la mécanique depuis 3 siècles. (Doctorat en génie industriel), Université Pierre Mendès France- École Nationale Supérieure de Génie Industriel, Grenoble.

Lavoisy, O. (2004). Illustration and Technical Know-How in Eighteenth-Century France. Journal of design history, 17(2), 141-162.

Le Masson du Parc. F. (2004). Pêches et pêcheurs du domaine maritime aquitain au XVIII ${ }^{\mathrm{e}}$ siècle. Amirauté de Bayonne. Saint-Quentin-de-Baron: Les éditions de l'Entre-deuxMers.

Le Masson du Parc. F. (2004). Pêches et pêcheurs du domaine maritime et des îles adjacentes de Saintonge, de l'Aulnis et du Poitou au XVIII ${ }^{\mathrm{e}}$ siècle. Saint-Quentin-de-Baron: Les éditions de l'Entre-deux-Mers.

Le Rond d'Alembert, J. (1751). Discours préliminaire. In D. Diderot \& J. D'Alembert (Éds.), Encyclopédie, ou Dictionnaire raisonné des sciences, des arts et des métiers. Paris: Briasson, David, Le Breton \& Durand.

Leontiev, A. (1984). Activité, conscience, personnalité. Moscou: Éditions du Progrès. 
Libert, L., \& Maucorps, A. (1969). Le ramendage des filets de pêche. Revue des Travaux de l'Institut des Pêches Maritimes, 32(2), 149-235.

Lettres patentes du Roy qui commettent le sieur Le Masson Du Parc, commissaire de la marine, pour faire l'inspection des pesches du poisson de mer sur les costes de Flandre, Picardie, Normandie et Bretagne (23 avril 1726).

Mafarette-Dayries, P. (2000). Lacadémie royale des sciences et les grandes commissions d'enquête et d'expertise à la fin de l'ancien régime Annales historiques de la Révolution française (320), 121-135.

Martinand, J.-L. (1994). La didactique des sciences et de la technologie et la formation des enseignants. AS$\operatorname{TER}(19), 61-75$.

Martinon-Torres, M. (2002). Chaîne opératoire: the concept and its application within the study of technology. Gallaecia(21), 29-43.

Mauss, M. (2004). Les techniques et la technologie. Revue du MAUSS, 1(23), 434-450.

Michaud, C. (2000, 12 mai). Duhamel du Monceau et l'Europe. Paper presented at the Colloque Duhamel du Monceau, Orléans.

Nonnon, É. (2007). Goody Jack. Pouvoirs et savoirs de l'écrit- Privat, Jean-Marie, Kara, Mohammed (coord.).La littéracie. Autour de Jack Goody. Revue française de pédagogie(161), 123-126.

Peaucelle, J.-L. (2007). Adam Smith et la division du travail. La naissance d'une idée fausse. Paris: L'Harmattan.

Perpillou, A. (1946). Pêches et pêcheurs de la Bretagne Atlantique. Annales de Géographie, 55(299), 200-204.

Picon, A. (1992). Gestes ouvriers, opérations et processus techniques. La vision du travail des encyclopédistes. Recherches sur Diderot et sur l'Encyclopédie(13), 131147.

Picon, A. (2001). Imaginaire de l'efficacité, pensée technique et imagination. Réseaux, 5(109), 18-50.

Pinault-Sorensen, M. (2000, 12 mai). La description des Arts et Métiers et le rôle de Duhamel du Monceau. Paper presented at the Colloque Duhamel du Monceau, Orléans.

Reuter, Y. (2006). À propos des usages de Goody en didactique. Éléments d'analyse et de discussion. Pratiques(131-132), 131-154.

Sensevy, G. (2001). Théories de l'action et action du professeur. In J.-M. Baudoin \& J. Friedrich (Éds.), Théories de l'action et éducation (p. 203-224). Bruxelles: De Boeck.

Sensevy, G. (2007). Des catégories pour décrire et comprendre l'action didactique. In G. Sensevy \& A. Mercier (Éds.), Agir ensemble. L'action didactique conjointe du professeur et des élèves (p. 13-45). Rennes: Presses Universitaires de Rennes.

Sensevy, G. (2011). Le sens du savoir: Éléments pour une théorie de l'action conjointe en didactique. Bruxelles: De Boeck.

Sensevy, G., Mercier, A., \& Schubauer-Leoni, M.-L. (2000). Vers un modèle de l'action didactique du professeur:
Caractérisation de certains éléments de cette action dans la course à 20. Recherches en Didactique des Mathématiques, 20(3), 263-304.

Sheridan, G. (2009). Louder than Words: Ways of Seeing Women Workers in Eighteenth-Century France. Lubbock: Texas Tech University Press.

Thibault, M. (2000, 12 mai). Duhamel du Monceau et la pêche. Paper presented at the Colloque Duhamel du Monceau, Orléans.

Vatin, F. (2004). Mauss et la technologie. Revue du MAUSS, 1(23), 418-433.

Vergnaud, G. (1994). Homomorphismes réel-représentation et signifié-signifiant- Exemples en mathématiques. Didaskalia(5), 25-34.

Vérillon, P., Leroux, P., \& Manneux, G. (2005). Activité productive et processus constructifs à l'école: les activités scolaires de production peuvent-elles être source de construction pour les élèves? ASTER(41), 3-26.

Vérin, H. (2000, 12 mai). Duhamel du Monceau et le monde des ingénieurs. Paper presented at the Colloque Duhamel du Monceau, Orléans. 